\title{
BMJ Open Rationale and design of GENEiUS: a prospective observational study on the genetic and environmental determinants of body mass index evolution in Canadian undergraduate students
}

\author{
Rita E Morassut, ${ }^{1}$ Christine Langlois, ${ }^{1}$ Akram Alyass, ${ }^{1}$ Adeola F Ishola, ${ }^{1}$ \\ Fereshteh T Yazdi, ${ }^{1}$ Alexandra J Mayhew, ${ }^{1}$ Hudson Reddon, ${ }^{1}$ James MacKillop, ${ }^{2}$ \\ Marie Pigeyre, ${ }^{1,3}$ David Meyre ${ }^{1,4}$
}

To cite: Morassut RE, Langlois C, Alyass A, et al. Rationale and design of GENEiUS: a prospective observational study on the genetic and environmental determinants of body mass index evolution in Canadian undergraduate students. BMJ Open 2017;7:e019365. doi:10.1136/ bmjopen-2017-019365

- Prepublication history and additional material for this paper are available online. To view these files, please visit the journal online (http://dx.doi. org/10.1136/bmjopen-2017019365).

Received 29 August 2017 Revised 6 October 2017 Accepted 31 0ctober 2017

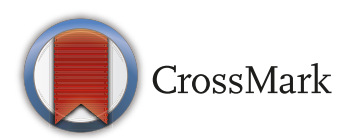

${ }^{1}$ Department of Health Research Methods, Evidence, and Impact, McMaster University, Hamilton, Canada

${ }^{2}$ Peter Boris Centre for Addictions Research, McMaster University/St. Joseph's Healthcare Hamilton, Hamilton, Ontario, Canada

${ }^{3}$ Department of Nutrition, University of Lille, CHRU Lille, Lille, France

${ }^{4}$ Department of Pathology and Molecular Medicine, McMaster University, Hamilton, Canada

Correspondence to

Dr David Meyre;

meyred@mcmaster.ca

\section{ABSTRACT}

Introduction Obesity is a global epidemic and is a risk factor for developing other comorbidities. Young adulthood is a critical period for body weight change and establishing healthy lifestyle behaviours. The 'Freshman 15 ' suggests that undergraduate students gain $15 \mathrm{lbs}$ $(6.8 \mathrm{~kg})$ during their first year of university, although evidence estimates a more modest weight gain of approximately 3-5 lbs (1.4-2.3 kg). Previous studies have only investigated weight change in the first year and do not study potential risk factors. Genetic and EnviroNmental Effects on weight in University Students (GENEiUS) is a prospective observational study which will investigate the environmental and biological determinants of weight change in undergraduate students over 4 years.

Methods and analysis The GENEiUS study will recruit 2500 multiethnic undergraduates aged 17-25 years at McMaster University at the start of their first year and will follow them every 6 months for 4 years. Primary outcomes are obesity traits: body mass index, waist circumference, waist-to-hip ratio, body fat mass and body fat percentage. The contribution of well-established and novel genetic variants for obesity traits and heritability values will be derived from whole-genome single-nucleotide polymorphism genotyping arrays. Civil status, age, sex, ethnicity, length of residence in Canada, religiosity, energy intake, physical activity, exercise motivation, electronic screen time, sleep patterns, history of assault, smoking status, alcohol consumption, medication and drug use, stress, impulsivity, body image perception, self-esteem, anxiety, eating disorders and depression will be investigated for their effect on obesity traits. The findings of the GENEiUS study will be used to help design obesity prevention programme in North American universities with multiethnic populations.

Ethics and dissemination Ethical approval of the study protocol has been obtained from the Hamilton Integrated Research Ethics Board. Study results will be disseminated through scientific publications, scholarly meetings, and collaborative meetings with university administration and student groups.
Strengths and limitations of this study

- This is the first adequately powered study to investigate genetic associations of weight change in undergraduate students.

- This is the first study to investigate an exhaustive number of potential risk factors for weight change in undergraduate students.

- While most studies investigating weight changes in undergraduates have taken place over 6-8 months, this study will follow students throughout their studies for 4 years.

- Validated questionnaires will be used to measure the majority of parameters in order to decrease participant burden and limit cost.

- This study is sufficiently powered to identify genetic and environmental predictors of weight change in undergraduate students; however, it is not powered to assess gene-environment interactions.

\section{INTRODUCTION}

Obesity in young adulthood

Obesity has become a global epidemic and affects one in five adults in Canada. ${ }^{12}$ This is of great concern to public health as obesity is a major risk factor for depression, osteoarthritis, type II diabetes, hypertension, cardiovascular disease and some cancers. ${ }^{34}$ The high prevalence, associated comorbidities and increased mortality make obesity a monumental economic burden accounting for $\$ 7.1$ billion in Canadian healthcare costs. ${ }^{5}$ Adolescence and young adulthood may be critical periods for the development of obesity as elevated body mass index (BMI) during this time is associated with obesity later in adulthood. ${ }^{6}$ When comparing the prevalence of obesity in Canadian adults from 2010 to 2014, the largest increase was 
observed among individuals aged 18-19 years. ${ }^{7}$ While education status is negatively correlated with BMI in the general population from high-income countries, young adults with higher education gain more weight and are more likely to be obese than those without university education in the USA. ${ }^{8-10}$

The 'Freshman 15 ' is a popular theory which suggests that university undergraduate students gain $15 \mathrm{lbs}$ $(6.8 \mathrm{~kg})$ during their first year of postsecondary education, although the average weight gain is estimated to be $3-5$ lbs $(1.4-2.3 \mathrm{~kg}) \cdot{ }^{11-13}$ It should be noted that these studies are limited by small sample sizes, high femaleto-male ratios, and may be susceptible to volunteer bias due to high dropout rates. Furthermore, sex-specific and ethnicity-specific subgroup analyses are scarce. Most studies are also only conducted over 1 year and there are limited and conflicting reports over weight change afterward. While one study suggests that students gain weight consistently, another describes stabilisation after the first year of undergraduate education. ${ }^{14}{ }^{15}$ Overall, students tend to weigh more in the fourth year compared with the first year and it is unclear whether this can be attributed to continued physical development and maturation or the accumulation of fat mass. ${ }^{16} 17$ The entire duration of undergraduate studies may therefore be a critical period for weight change in young adults and this study will provide insight into the genetic and environmental determinants of weight change among this population.

\section{Predictors of undergraduate weight change}

The aetiology of obesity is complex and depends on a variety of biological, environmental, lifestyle and socioeconomic factors. ${ }^{18} 19$ There is limited evidence on factors influencing weight in undergraduate students. Excessive consumption of calorie-dense foods, the number of meals consumed during the evening and weekends and recent dieting have all been associated with weight gain in undergraduate students. ${ }^{20-22}$ Evidence suggests that a lack of healthy food options on campus and buffet-style meal plan design may facilitate the development of unhealthy eating patterns among students. ${ }^{2324}$ Further, the increased expenses of university tuition and living away from home limit a student's budget to purchase healthy foods which often cost more. ${ }^{23}{ }^{24}$ First year students tend to make unhealthier food choices and increase alcohol consumption as each academic semester progresses, especially near examination time. ${ }^{25-28}$ These poor eating habits coupled with decreased physical activity and increased sedentary behaviour from internet usage may partially explain the mean weight gain of $3-5$ pounds in the first year. ${ }^{29}$ Their low motivation to exercise may also be detrimental as greater motivation promotes weight loss in both the short and long term. ${ }^{24} 30$

Undergraduate students sleep less and more erratically than high school students. ${ }^{27}$ Sleep and BMI have a U-shaped relationship such that short and long sleep durations are associated with elevated BMI. ${ }^{31}$ In undergraduates, a decrease in the quantity but not the quality of sleep is related to weight gain. ${ }^{20}$ Students are also subject to greater stress as they transition to undergraduate studies due to an increased academic workload and by living away from home and away from social support systems. Stress has previously been associated with both weight gain and weight loss in undergraduate students. ${ }^{28}$ Interestingly, BMI may moderate the effect of stress on weight change in students as high stress has been associated with weight gain among those with high baseline BMI and weight loss in those with low baseline BMI. ${ }^{32}$ Weight may also be influenced by adverse experiences. History of sexual assault has been linked to obesity and elevated BMI in some studies. ${ }^{33} 34$ This is of particular concern to university students as $14 \%-44 \%$ report experiencing some form of sexual assault since starting undergraduate studies. ${ }^{35}$

Undergraduate students have described lack of self-control as a barrier to making healthy dietary choices. ${ }^{23}$ Impulsivity in the context of weight maintenance, as measured by delayed discounting, reflects preferences for small immediate pleasures compared with larger rewards later in life. Steeper delayed discounting, indicating more impulsive choices, has been associated with obesity, especially in adolescents. ${ }^{36}$ Further, impulsive personality traits have been positively associated with BMI in university students indirectly through compulsive eating behaviours. $^{37}$

Psychiatric conditions may also impact the weight of undergraduate students. Bulimia nervosa and binge eating disorder have been associated with elevated BMI. ${ }^{38}$ Young adults are especially at risk for pathological eating since the prevalence of eating disorders appears to increase from $0.3 \%$ in adolescence to $1 \%$ in young adulthood. ${ }^{39}$ Although there is weak evidence, disordered eating habits have been associated with overweight and obesity in university students. ${ }^{40}$ Further, among female but not male students, weight gain has been associated with greater disordered eating behaviour over 4 years. ${ }^{14}$ Both depression and anxiety have been associated with obesity. ${ }^{41}$ These conditions are of major concern on university campuses since it is estimated that $18.3 \%$ and $14.7 \%$ of Canadian students have anxiety and depression, respectively. ${ }^{42}$ Limited studies show that depression appears to be associated with obesity in male, but not female, undergraduate students. ${ }^{14} 43$ Finally, other psychological factors including body image dissatisfaction and low self-esteem have been associated with overweight and obesity in adolescents. ${ }^{44} \mathrm{Few}$ studies have investigated these in undergraduate students, and there are conflicting reports whether they are related to weight change. $^{4546}$

Among those aged 15-24 years in Canada, approximately $71 \%$ report a religious affiliation. ${ }^{47}$ Religiosity has been associated with healthy lifestyle behaviours in adolescents. ${ }^{48}$ While religiosity has been shown to have protective effects on cardiometabolic traits like diabetes and hypertension, it has been associated with elevated BMI. $^{49}{ }^{50}$ One study found increased incidence of obesity 
in religious young adults. ${ }^{51}$ Therefore, the effect of religiosity on weight in undergraduates warrants further investigation. Further, birthplace and length of residence may also contribute to weight change in undergraduate students. In the USA, foreign-born immigrants tend to have lower BMI than those born in the USA, yet experience an increase in BMI with length of residence. ${ }^{52}$

Genome-wide association studies (GWAS) have identified many single-nucleotide polymorphisms (SNPs) associated with BMI, waist-to-hip ratio (WHR) and body fat percentage $(\% \mathrm{BF})$; however, these markers are not specific to university students. ${ }^{53-55}$ To the best of our knowledge, only one study from the UK has explored the genetic causes of weight gain in university. It found that FTO SNP rs9939609, the most important genetic contributor to obesity in European populations, showed a nominal association with weight gain at the end of the first academic year. ${ }^{56}$ However, the study suffered from several limitations: high dropout rate $(78 \%)$, low statistical power $(n=310)$ and limited follow-up duration.

To design effective obesity prevention programme in universities, a better understanding of its causes in undergraduate students is needed. The Genetic and EnviroNmental Effects on weight in University Students (GENEiUS) study will investigate the genetic and environmental determinants of obesity trait evolution in undergraduate students at McMaster University (Hamilton, Ontario, Canada) over the 4-year course of their studies.

\section{Study objectives}

The primary objectives of this study are to (1) describe BMI level and change over 4years, (2) investigate the heritability and genetic determinants of BMI level and change and (3) investigate the social, cultural, lifestyle and environmental determinants of BMI level and change in undergraduate students. Secondary objectives of the GENEiUS study include the investigation of genetic and environmental determinants of additional obesity traits (waist circumference, WHR, \% BF and body fat mass) and endophenotypes.

\section{METHODS AND STUDY DESIGN}

\section{Participant selection and recruitment}

The GENEiUS study is a longitudinal prospective observational study in Hamilton, Ontario. Undergraduate students from McMaster University will be followed every 6 months over 4 years beginning in September of their first year of study. First year students enrolled at McMaster University between the ages of 17 and 25 years are eligible to participate in the study. ${ }^{57}$ Individuals who are pregnant, have given birth or have a medical condition which can impact BMI for a long period of time (eg, bariatric surgery, immobilisation from injury, etc) will be excluded from the study.

Beginning in September 2015, first year students are invited to participate from September and October of each year. Recruitment will be conducted via advertising through presentations, information tables, posters, flyers and social media. Interested students will be given detailed information about the study in person, via email or by phone. Approximately 500 participants will be recruited per year for about 5 years until a goal of 2500 participants is reached. Students will be evaluated for eligibility to participate at the baseline visit and provide informed consent prior to enrolment. Participants will be reimbursed with a gift card on completion of each study appointment.

\section{Data collection}

Participants will be assessed every 6 months for 4 years, once in September or October and again in March or April of each academic year (table 1). At the baseline visit only, a saliva sample will be obtained in addition to information on age, sex, ethnicity, birthplace and country of residence history. Weight, height, waist circumference (WC), hip circumference (HC), \% BF and body fat mass (BFM) will be measured at baseline and all follow-up appointments. Socioeconomic status, civil status, sexual orientation, medical history, medication and drug use, smoking status, energy intake, meal plan type, alcohol consumption, physical activity, electronic screen time, method commuting to campus, living status on or off campus, sleep health, stress, history of abuse, impulsivity, eating disorders, body dissatisfaction, self-esteem, depression, anxiety and religiosity will be measured at all time-points using online, self-reported questionnaires. Participants will complete their questionnaires and one food recall at their baseline visit with instruction from a research assistant. After receiving this initial training, follow-up questionnaires and food recalls will be completed online outside of study appointments at the participant's leisure to minimise burden. All questionnaires and food recalls will be completed within 3 weeks of a participant's study appointment.

\section{Outcomes}

The obesity trait outcome variables are BMI, WC, WHR, $\% \mathrm{BF}$ and BFM. Trained research personnel will perform all anthropometric measurements in duplicate to reduce intrarater variability. Participants will wear light clothing and remove shoes before being weighed. Weight will be measured to the nearest $0.1 \mathrm{~kg}$ using a digital scale (Seca, Hamburg, Germany). Height will be measured to the nearest $0.1 \mathrm{~cm}$ using a portable stadiometer (Seca 225, Hamburg, Germany). WC will be measured after a normal exhalation at the midpoint of the last palpable rib and the superior portion of the iliac crest to the nearest $0.1 \mathrm{~cm}$ and $\mathrm{HC}$ will be measured at the widest part of the buttocks to the nearest $0.1 \mathrm{~cm}$ using a stretch-resistant tape measure, as previously described by the WHO. ${ }^{58}$ WHR will be calculated as WC divided by HC. BMI (kg/ $\mathrm{m}^{2}$ ) will be calculated by dividing weight by squared height. Bioelectric impedance analysis (BIA) will be used to assess BFM to the nearest $0.1 \mathrm{~kg}$ and $\% \mathrm{BF}$ to the nearest $0.1 \%$. Participants will remove jewellery before stepping 


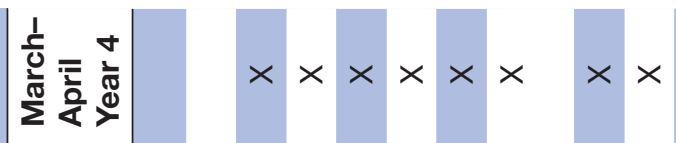

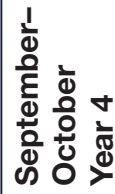

$$
\times \times \times \times \times \times \times
$$

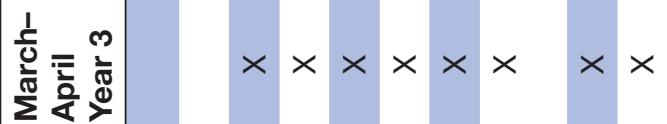

일

焉高。

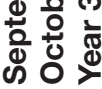

$\times \times \times \times \times \times \times$

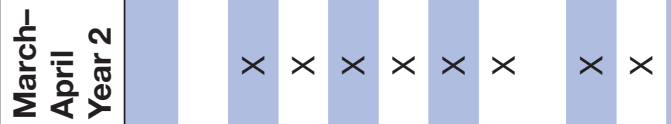

$\frac{1}{\Phi}$

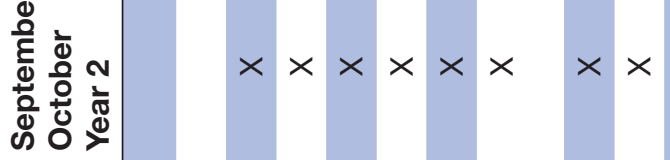

皇

$\times \times \times \times \times \times \times \times \times \times \times$

$\times \times \times \times \times \times \times \times \times \times \times$

$x \times \times \times \times \times \times \times \times \times \times$

$\frac{1}{d}$

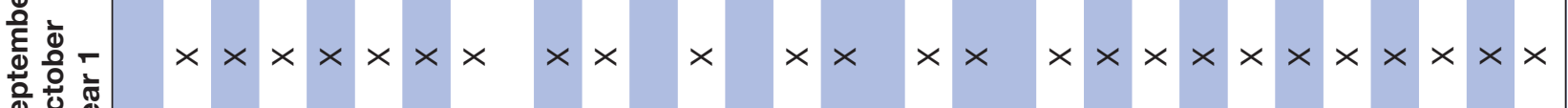

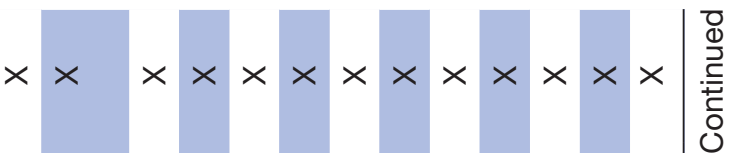

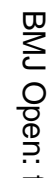

$\times \times \times \times \times \times \times \times \times$

$$
\times
$$$$
\times \times \times \times \times \times \times \times \times
$$

$\times \times \times \times \times \times \times \times \times$

官
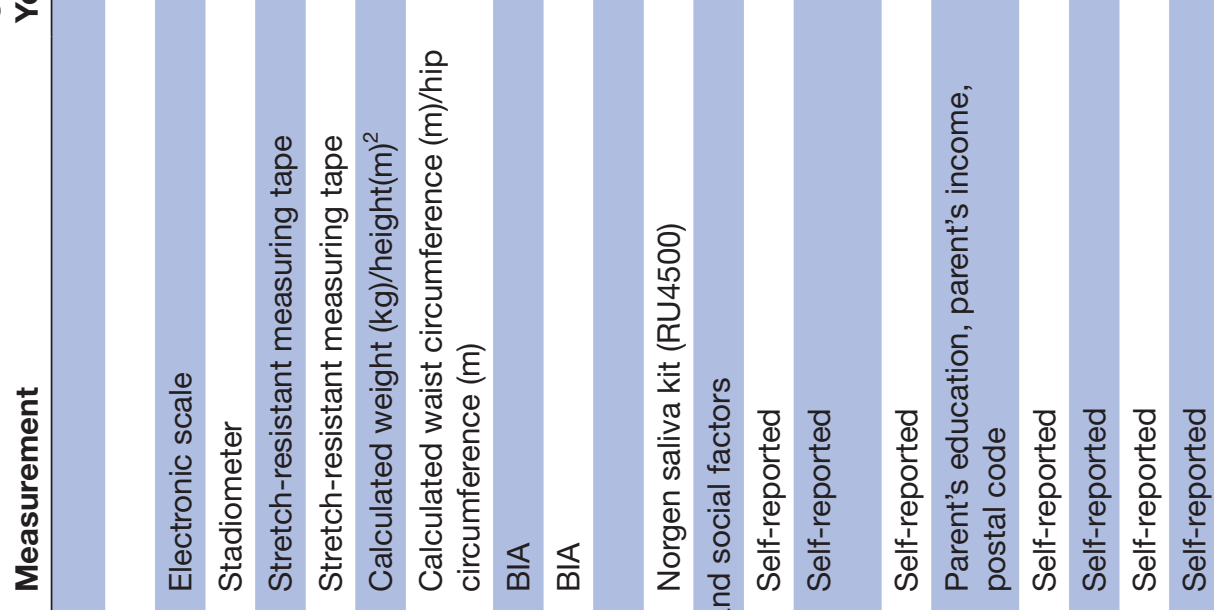

莡

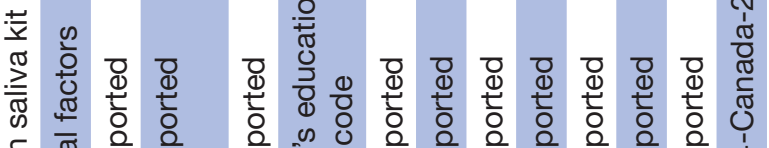




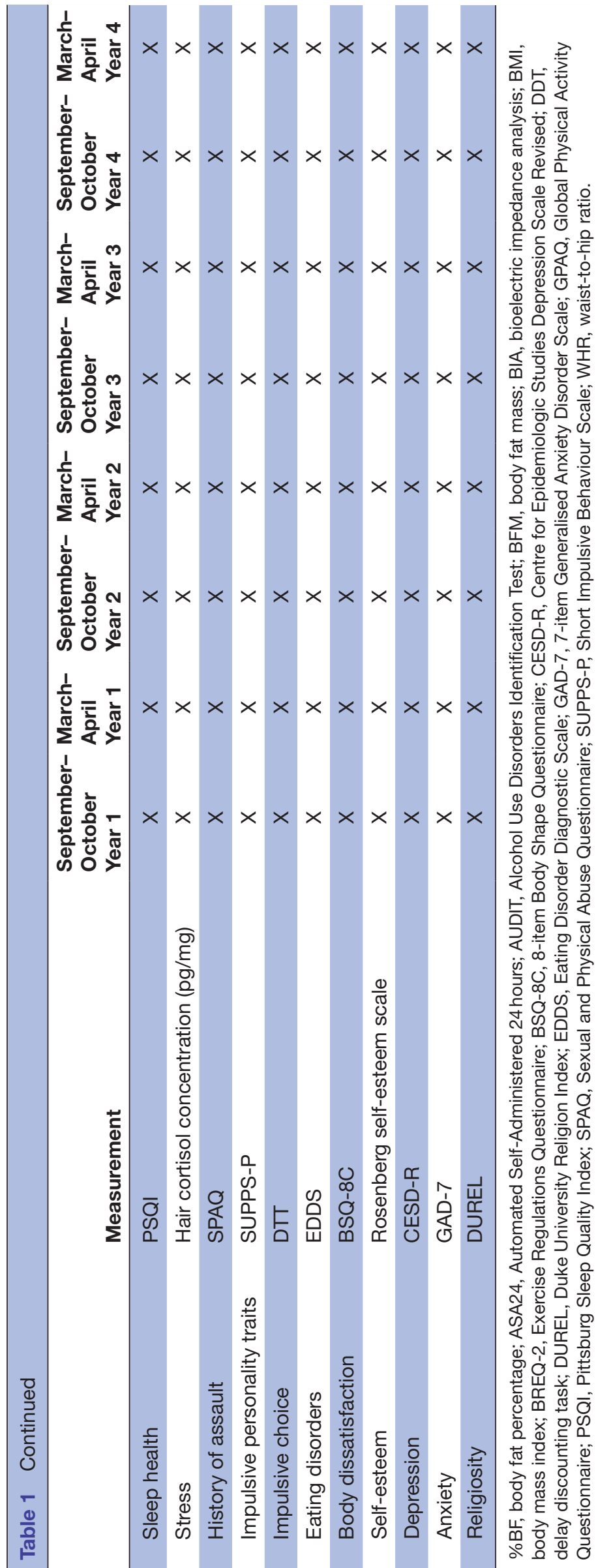


barefoot onto the BIA device (Tanita SC-331S, Arlington Heights, Illinois, USA).

\section{Predictor variables}

A variety of genetic and environmental predictors of weight will be measured. Potential risk factors for obesity including age, sex, ethnicity, socioeconomic status, civil status, sexual orientation, smoking status, medical conditions, medication or drug use, living status on or off campus, method of commuting to campus, meal plan type (of the several meal plan choices offered both on and off residence), time spent using electronic devices, birthplace and history of country residence will be evaluated through online, self-reported questionnaires.

\section{DNA extraction, whole-genome genotyping and whole-exome sequencing}

DNA will be collected from a saliva sample. Saliva will be collected and preserved using the Norgen saliva collection kit (RU4500, Norgen Biotek Corporation, Thorlord, Canada) as per the manufacturer's instructions and stored at room temperature. Before purification, saliva samples will be incubated overnight at $50^{\circ} \mathrm{C}$. DNA will be purified from $4 \mathrm{~mL}$ of preserved saliva using the Chemagen 500 MSM I (PerkinElmer chemagen Technologie $\mathrm{GmbH}$, Baesweiler, Germany) as per the manufacturer's instructions. Two types of genetic experiments will be performed in the present study using Illumina microarray genotyping and next-generation sequencing platforms at the McMaster Genome Facility. Genotyping of whole-genome SNP arrays for each participant will be done to study the association of common and low-frequency genetic variants (minor allele frequency $>0.005$ ) with obesity traits. We plan to use the Illumina Infinium Multi-Ethnic Global-8 microarray because it combines a relatively high density of SNPs (1.7 million) with an SNP tagging strategy adapted to ethnically diverse populations. Whole-exome sequencing (WES) will be used to study the association of rare coding variants with obesity traits. WES will be performed on a subset of participants who have extreme decreases in BMI ( $\leq 10$ th percentile), maintain BMI (45th to 55 th percentile) and extreme increases in BMI ( $\geq 90$ th percentile) between baseline and 1 year post baseline. We also plan to use the Illumina TrueSeq Exome kit for DNA library preparation and HiSeq 1500 for DNA sequencing, as per the manufacturer's instructions.

\section{Energy intake}

Energy intake will be assessed with a 3-day 24-hour food recall, which was selected for its validity, reliability and minimal participant burden. ${ }^{59}$ Participants will recall their energy intake from 2 weekdays and 1 weekend day using the 2016 Canadian adaptation of the Automated Self-Administered 24-hour Recall. ${ }^{60}$ Three-day total energy intake will be averaged to give a mean value per day in kcal. Values for nutrient intake including total carbohydrates $(\mathrm{g})$, protein $(\mathrm{g})$ and fats $(\mathrm{g})$, including saturated fat, monounsaturated fat and polyunsaturated fat will be reported.

\section{Alcohol consumption}

The self-reported version of the Alcohol Use Disorders Identification Test (AUDIT) will be used as a validated measure of alcohol consumption. ${ }^{61}$ The AUDIT is a 10-item questionnaire with each question rated on a 5-point scale from 0 to 4 and summed to give a total global score out of 40. Higher scores indicate greater risk of dangerous drinking habits. A score of 8 or more indicates harmful alcohol use with possible alcohol dependence.

\section{Physical activity}

Physical activity will be assessed using a 16-item, self-reported version of the Global Physical Activity Questionnaire (GPAQ). The GPAQ was selected for its use in broad populations and its ability to assess physical activity and sedentary time at work, for recreation and during transportation. $^{62}$ Although the GPAQ is traditionally delivered in-person or via telephone interview, self-reported administration has been shown to be a comparable alternative and the self-reported version will be used in this study for its feasibility. ${ }^{63}$ The GPAQ will be scored by calculating Total Metabolic Equivalent of Task minutes per week as previously described by the WHO. ${ }^{64}$

\section{Exercise motivation}

The exercise regulations questionnaire is a 19-item, self-reported questionnaire which measures motivation towards exercising in five domains: external regulation, intrinsic regulation, identification, introjection and motivation. ${ }^{65}$ Each item is scored on a 5-point scale from 0 (not true for me) to 4 (very true for me). The mean score of each subscale will be calculated and used in analysis. Higher scores indicate greater motivation.

\section{Sleep}

Sleep health will be measured by the Pittsburg Sleep Quality Index (PSQI) as a reliable measure for sleep quality in university students. ${ }^{66} 67$ The PSQI is a 24-item, self-reported questionnaire which measures seven domains of sleep health over the past month: sleep quality, sleep latency, sleep duration, habitual sleep efficiency, sleep disturbances, use of sleep medications and daytime dysfunction. Each domain is scored on a 4-point scale from 0 (not during the past month) to 3 (three or more times per week) and summed to give a total global score out of 21. Higher scores indicate poorer sleep quality.

\section{Stress}

Hair cortisol concentration $(\mathrm{pg} / \mathrm{mg}$ ) will be measured as a biochemical marker for exposure to physical stressors and ongoing chronic stress. ${ }^{68}$ Hair strands will be cut as close to the scalp as possible from the posterior vertex position. At least $10 \mathrm{mg}$ of hair from the most proximal $3 \mathrm{~cm}$, representing the last 3 months of hair growth, will be analysed. ${ }^{69}$ Cortisol will be extracted as previously described and measured using ELISA. ${ }^{70}$ 


\section{History of abuse}

The Sexual and Physical Abuse Questionnaire (SPAQ) is a 9-item, self-reported questionnaire and will be used as a validated measure of physical and sexual abuse. ${ }^{71}$ It assesses abuse at four time points: $(1)<6$ years old, (2) $>6$ but $<12$ years old, (3) $>12$ but $<16$ years old and (4) $>16$ years old. Positive answers indicate a history of abuse. Due to the potentially triggering nature of this questionnaire, participants will be provided with contact information for local legal and support resources at the time of data collection.

\section{Impulsivity}

To measure impulsive personality traits, the Short UPPS-P Impulsive Behaviour Scale will be used and was selected for its use in university students. ${ }^{72}{ }^{73}$ It is a 20 -item, self-reported questionnaire with subscales to measure negative urgency, positive urgency, lack of perseverance, lack of premeditation and sensation seeking. Items are scored on a 4-point scale from 1 (strongly agree) to 4 (strongly disagree) with higher scores indicating more impulsive behaviour. Mean scores from each subscale will be calculated and summed to give a total global score from 5 to 20 .

A computer-administered delay discounting task (DDT) will be used to measure impulsive choice. ${ }^{74}$ This is a 5 -item task, whereby participants are asked to choose between an immediate reward of $\$ 100$ or a delayed reward of $\$ 1000$. Monetary values will be held constant, while temporal delays will be adjusted up or down, ranging from 1 hour to 25 years, depending on the choice made by each participant. This task will result in 32 potential Effective Delay $50 \%\left(\mathrm{ED}_{50}\right)$ scores approximately evenly spaced on a logarithmic scale. Temporal discounting rates, $k$ values, will be generated for each participant by calculating the inverse of $\mathrm{ED}_{50}$. Higher $k$ values reflect greater impulsivity.

\section{Eating disorders}

Eating disorders will be evaluated using the Eating Disorder Diagnostic Scale (EDDS) developed for use in community populations. ${ }^{75}$ The EDDS is a 22-item, self-reported questionnaire which assesses Diagnostic and Statistical Manual of Mental Disorders, Fifth Edition (DSM-5) criteria for diagnosing anorexia nervosa, bulimia nervosa and binge-eating disorder in respondents. It will be scored as previously described. ${ }^{75}$ Scores will be analysed dichotomously by eating disorder diagnosis and continuously by total global score.

\section{Body dissatisfaction}

Concern about body image will be measured using the 8-item Body Shape Questionnaire (BSQ-8C) for its use in community populations. ${ }^{76} 77$ The BSQ-8 is a self-reported questionnaire which assesses body shape concerns over the last 4 weeks on a 6-point scale from 1 (never) to 6 (always). Higher scores indicate greater body dissatisfaction.

\section{Self-esteem}

Self-worth will be measured using the Rosenberg selfesteem scale for its wide use in general populations. ${ }^{78}$ It is a self-reported questionnaire which measures self-esteem on a 4-point scale from 1 (strongly disagree) to 4 (strongly agree). Higher scores indicate higher self-esteem.

\section{Depression}

Depressive symptoms will be evaluated by the Centre for Epidemiological Studies Depression Scale Revised (CESD-R) for its validated use in university students. ${ }^{79} 80$ The CESD-R is a 20-item, self-reported questionnaire with subscales to measure sadness, loss of interest, appetite, sleep, thinking and concentration, guilt, fatigue, agitation and suicidal ideation during the past week. The CESD-R is scored using a frequency 5-point scale for each item from 0 (not at all or less than 1 day) to 4 (nearly every day for 2 weeks). Higher scores indicate greater depressive symptoms. A cumulative score of at least 16 out of 60 indicates risk of clinical depression. A major depressive episode is characterised by loss of interest or sadness nearly every day for the past 2 weeks, and symptoms in another four DSM symptom groups occurring nearly every day for the past 2 weeks.

\section{Anxiety}

The 7-item Generalised Anxiety Disorder Scale (GAD-7) is a valid measure of anxiety. ${ }^{81}$ The GAD-7 is a self-reported questionnaire which assesses anxious symptoms in the last 2 weeks on a 4-point scale from 0 (Not at all) to 3 (Nearly every day). Higher scores indicate greater anxiety. Scores of 10 or greater indicate risk of an anxiety disorder.

\section{Religiosity}

Participants will be asked to self-report their religious affiliation as Christian, Jewish, Muslim, Hindu, Buddhist, Sikh, Traditional (aboriginal) spirituality or No religion. ${ }^{47}$ Religiosity will be measured by the Duke University Religion Index (DUREL) as a widely used, validated tool for assessing religious involvement. ${ }^{82} 83$ The DUREL is a 5-item, self-reported questionnaire with subscales to measure organisational religious association, non-organisational religious association and intrinsic religiosity. Items measuring organisational and non-organisational religious association are assessed on a 6-point scale from 1 to 6 with higher scores reflecting more frequent religious attendance. Items measuring intrinsic religiosity are assessed on a 5-point scale from 1 (Definitely not true) to 5 (Definitely true of me). Individual subscales scores will be summed and analysed separately.

\section{Sample size calculation}

Most previous studies investigating weight change in undergraduate students have used sample sizes between 75 and 200 participants. ${ }^{11}$ With a sample size of 2000 , there is at least $80 \%$ power to detect effect sizes of $0.8 \mathrm{~kg} /$ $\mathrm{m}^{2}$ across a range of allele frequencies at the 5\% significance level for BMI level (table 2). For reference, the risk allele of FTO increases BMI level by approximately $0.4 \mathrm{~kg} /$ $\mathrm{m}^{2}$ and has a minor allele frequency of 0.4 in Europeans, although these differ by ethnicity. ${ }^{84}$ For BMI change, there is at least $80 \%$ power to detect effect sizes of $0.1 \mathrm{~kg} / \mathrm{m}^{2}$ 
Table 2 Sample sizes needed in a cohort design to detect significant associations of genetic variants with BMI level with $80 \%$ power and a two-sided $\mathrm{P}$ of 0.05 by beta coefficient and allele frequency for risk allele

\begin{tabular}{lrrrrrr}
\hline \multicolumn{7}{c}{ Minor allele frequency } \\
\hline Allelic beta & $\mathbf{0 . 0 1}$ & $\mathbf{0 . 0 5}$ & $\mathbf{0 . 1}$ & $\mathbf{0 . 2}$ & $\mathbf{0 . 3}$ & $\mathbf{0 . 4}$ \\
\hline 0.10 & 513741 & 107071 & 56508 & 31784 & 24215 & 21188 \\
0.20 & 128432 & 26765 & 14112 & 7943 & 6051 & 5294 \\
0.30 & 57079 & 11893 & 6275 & 3528 & 2687 & 2351 \\
0.40 & 32105 & 6688 & 3528 & 1983 & 1510 & 1321 \\
0.50 & 20546 & 4279 & 2257 & 1268 & 965 & 844 \\
0.60 & 14267 & 2970 & 1566 & 879 & 669 & 585 \\
0.70 & 10481 & 2181 & 1149 & 645 & 490 & 429 \\
0.80 & 8023 & 1669 & 879 & 493 & 374 & 327 \\
0.90 & 6339 & 1316 & 694 & 389 & 295 & 258 \\
1.00 & 5134 & 1067 & 561 & 314 & 238 & 208 \\
1.50 & 2279 & 472 & 257 & 137 & 104 & 90 \\
2.00 & 1280 & 264 & 137 & 75 & 57 & 49 \\
2.50 & 818 & 167 & 86 & 47 & 35 & 30 \\
3.00 & 567 & 115 & 59 & 31 & 23 & 19 \\
3.50 & 415 & 83 & 42 & 22 & 16 & 13 \\
\hline
\end{tabular}

BMI, body mass index.

Table 3 Sample sizes needed in a cohort design to detect significant associations of genetic variants with BMI change with a power of $80 \%$ and a two-sided P of 0.05 by beta coefficient and allele frequency for risk allele

\section{Minor allele frequency}

\begin{tabular}{lrrrrrr}
\hline Allelic beta & \multicolumn{1}{l}{$\mathbf{0 . 0 1}$} & \multicolumn{1}{l}{$\mathbf{0 . 0 5}$} & \multicolumn{1}{l}{$\mathbf{0 . 1}$} & $\mathbf{0 . 2}$ & $\mathbf{0 . 3}$ & $\mathbf{0 . 4}$ \\
\hline 0.01 & $>1000000$ & 669216 & 353196 & 198671 & 151367 & 132446 \\
0.02 & 802722 & 167301 & 88296 & 49665 & 37839 & 33109 \\
0.03 & 356763 & 74354 & 39240 & 22071 & 16815 & 14713 \\
0.04 & 200678 & 41822 & 22071 & 12413 & 9457 & 8274 \\
0.05 & 128432 & 26765 & 14124 & 7943 & 6051 & 5294 \\
0.06 & 89188 & 18586 & 9807 & 5515 & 4201 & 3675 \\
0.07 & 65525 & 13654 & 7204 & 4051 & 3085 & 2699 \\
0.08 & 50166 & 10453 & 5515 & 3100 & 2361 & 2066 \\
0.09 & 39637 & 8258 & 4357 & 2449 & 1865 & 1631 \\
0.10 & 32105 & 6688 & 3528 & 1983 & 1510 & 1321 \\
0.20 & 8023 & 1669 & 879 & 493 & 374 & 327 \\
0.30 & 3564 & 740 & 389 & 217 & 164 & 143 \\
0.40 & 2003 & 414 & 217 & 120 & 91 & 79 \\
0.50 & 1280 & 264 & 137 & 75 & 57 & 49 \\
0.60 & 888 & 182 & 94 & 51 & 38 & 33 \\
0.70 & 651 & 133 & 68 & 36 & 27 & 23 \\
0.80 & 498 & 101 & 51 & 27 & 19 & 16 \\
0.90 & 392 & 79 & 40 & 20 & 14 & 12 \\
\hline 1.00 & 317 & 63 & 31 & 16 & 11 & 9 \\
\hline
\end{tabular}

BMI, body mass index. 
across a range of allele frequencies at the $5 \%$ level of significance (table 3). To account for a $20 \%$ dropout rate, an additional 500 individuals will be recruited for a final sample size of $2500 .{ }^{13}$ Dropout is defined as missing two consecutive appointments since it reflects one full academic year. Sample size estimates for GWAS, exomewide association study for rare variants and associations for binary environmental traits are included for reference for BMI level (see online supplementary tables 1-3) and BMI change (see online supplementary tables 4-6). Our targeted sample size will provide reasonable to good statistical power for these analyses.

\section{Statistical methods}

Descriptive statistics at baseline will illustrate the distribution of sex, age, sexual orientation, civil status, socioeconomic status, ethnicity, proportion born in Canada, distribution of economic status of birth country (high/ middle/low income), residence status on/off campus, meal plan type (yes/no, high/medium/low cost), total and carbohydrate/fat/protein energy intake, alcohol consumption, physical activity, exercise motivation, electronic screen time, sleep quality, hair cortisol concentrations, history of sexual assault, impulsive personality traits, impulsive choice, disordered eating, body dissatisfaction, self-esteem, depressive symptoms, anxiety, religiosity, religious affiliation, BMI, WC, WHR, BFM and $\% \mathrm{BF}$. Continuous variables will be reported by mean and SD. Categorical variables will be reported by count and percentage. Subgroup analyses (eg, ethnicity) on obesity outcomes will be performed if there is a significant interaction between the predictor and the subgroup factor.

\section{Analysis of environmental risk factors}

The effect of potential environmental risk factors on obesity traits (ie, BMI, WC, WHR, \%BF and BFM) at baseline will be tested using linear regression models with adjustments for age, sex and ethnicity. For variables such as sleep health which are evaluated by scaled questionnaires, the cumulative global score will be used in the analyses to increase power. The effect of each potential environmental risk factor at baseline and during follow-up on the evolution of obesity traits at 1 year (interim) and 3.5-year (final) post baseline visit will be tested using mixed effects linear regression models with adjustment for age, sex, ethnicity and baseline BMI. Multivariate hierarchical stepwise mixed regression models will be used to assess the independent effect of each environmental risk factor on obesity trait trajectories. Collinearity and sensitivity analyses will be conducted to ensure stability of the model. Mediation and interaction analyses will be conducted when there is scientific rationale or biological plausibility for a relationship between variables.

\section{Analysis of genetic risk factors}

All analyses will investigate associations with genetic variants at baseline, 1 year and 3.5 years post baseline. Heritability estimates for obesity trait level and change will be calculated. GWAS will be performed to identify genetic variants associated with obesity trait level and change. Significance of genetic variants will be tested at the genome-wide level of significance $\left(\mathrm{P}<5 \times 10^{-8}\right)$. Genebased association tests for SNPs will also be performed for obesity traits. WES analysis will be performed to identify rare genetic coding variants associated with obesity trait level and change. Significance of SNPs and exonic variants will be tested at the gene-based level of significance $\left(\mathrm{P}<2.5 \times 10^{-6}\right){ }^{85}$ Hypothesis-free whole-genome approaches (GWAS and WES) do not have the power to detect all the genetic variants contributing to obesity because of the multiple testing burden from the Bonferroni correction. ${ }^{86}{ }^{87}$ Testing a subset of strong candidate genes or SNPs reduces the number of tests conducted and has the potential to lead to the identification of obesity genes missed by conventional approaches. ${ }^{88}$ These strategies will be conducted in GENEiUS. Genome-wide Complex Trait Analysis will be used to assess the heritability of obesity traits and change in obesity traits from SNP variants previously identified by GWAS. ${ }^{89}$ The PLINK software will be used for GWAS. ${ }^{90}$ Autosomal and X chromosome SNPs will be imputed using the all ancestry 1000 Genomes Project reference panel and the miniMAC or IMPUTE2 software. ${ }^{91} 92$ Gene-based association of SNPs with obesity trait level and change will be assessed using VEGAS2 software ${ }^{93}$ Variant annotation and filtering from whole-exome sequences will be performed using the KGGSEQ framework. ${ }^{94}$

The association of well-established GWAS SNPs for BMI, WC, WHR and \%BF, or a combination of pathogenic mutations in previously identified monogenic genes, with obesity trait level and change at baseline will be performed using linear regression models with adjustment of age, sex and ethnicity/population structure ${ }^{53-55}$ The self-reported ethnicity will be verified using principal component analysis from the EIGENSTRAT software. ${ }^{95}$ Adjustment for ethnicity/population structure will be achieved by adding the 10 first principal components of EIGENSTRAT analysis in the regression model. A P value threshold of 0.05 will be considered significant given the high prior likelihood of association. SNP variants will be analysed individually and cumulatively as a gene score using additive, dominant and recessive modes of inheritance. An unweighted gene score, which estimates the effect of all obesity susceptibility genetic variants on obesity traits, will be calculated by summing the alleles positively correlated with individual obesity traits. ${ }^{96}$ Missing genotype values will be imputed using the method of the mean in the calculation of the gene score. This imputation will be performed for each SNP individually using the arithmetic average of the coded genotypes observed for all the successfully genotyped individuals in each ethnic group. An allelic burden test of functional mutations for previously identified obesity genes will be conducted to investigate the cumulative effect obesity genes mutations on obesity trait level and change. All models will be adjusted for age, sex and ethnicity/population structure. Interim 
and final analyses will also be conducted to determine the effect of predisposing obesity genetic variants on individual obesity traits and changes in obesity traits with adjustment for age, sex, ethnicity and baseline BMI.

\section{Prediction models of weight change}

Models will be created to assess the utility of genetic variants and environmental factors on predicting weight status. Support vector machine learning models will be used to determine the optimal combination of predictive SNPs for incident occurrence of overweight and/or obesity. ${ }^{97}$ Receiver operating characteristic curve and net reclassification improvement analyses will include environmental and genetic factors to estimate their ability to predict overweight, overweight/obese or obese status in undergraduate students. ${ }^{98}$ Optimal predictive values for continuous environmental traits will be determined by polychotomisation as previously described. ${ }^{100}$ Internal validation of the prediction models will involve trainingtesting split methods. ${ }^{101}$

\section{DISCUSSION}

Weight changes occurring in young adulthood may be important for the development of chronic obesity. ${ }^{6}$ However, this age group has been poorly studied. The GENEiUS study is the first adequately powered observational study design aiming to investigate genetic and environmental associations of weight change in undergraduate students. The majority of past studies investigating undergraduate weight gain have only followed students until the end of their first academic year (ie, 6-8 months); therefore, little is known about weight changes throughout undergraduate studies. ${ }^{11}$ The GENEiUS study aims to fill this knowledge gap by following students prospectively over their first 4 years of undergraduate study. The longitudinal nature of GENEiUS will permit the study of temporal relationships between obesity risk factors and strengthen the ability to make causal inferences regarding determinants of undergraduate weight change. This study includes the measurement of many well-established risk factors for obesity such as energy intake, physical exercise and sleep duration. ${ }^{102}$ GENEiUS will also investigate novel potential risk factors like exercise motivation, history of abuse, religiosity, stress measured by hair cortisol and the effect of immigration. Only few studies in undergraduate students have included the measurement of WHR, \%BF or BFM. ${ }^{11}$ Since BMI may be a biased estimate of body fat, the inclusion of these measures in GENEiUS will provide a better estimation of adiposity. ${ }^{103}$ While it may be argued that measurement of additional cardiometabolic outcomes (eg, type II diabetes, hypertension, dyslipidemia, fatty liver, atherosclerosis) may be important, these comorbidities are mainly driven by obesity in young adults at the population level. ${ }^{104-106}$ For this reason, evolution of obesity-related traits will be our main focus. The heterogeneous nature of the sample population consisting of multiethnic students from a variety of undergraduate programmes will also help to increase the generalisability of findings in North America and perhaps identify ethnic-specific effects.

While the GENEiUS study design has many strengths in its design, it also has limitations. First, it is restricted to measurement of only two time points each year from September to October and from March to April, without inclusion of a summer assessment. Since few students remain on campus during this period, a summer time point will be avoided. Further, while the great use of validated questionnaires will provide strong estimates for various environmental factors, many of these do not represent the criterion measure for their respective traits. For instance, the GPAQ overestimates moderate-to-vigorous physical activity when compared with accelerometer data. ${ }^{107}$ These short, validated questionnaires delivered online were selected by balancing accuracy and feasibility given the large sample size of the study. Although dual-energy X-ray absorptiometry is a more precise measure of adiposity, BIA will be used in the present study to increase access to the equipment, decrease participant burden and limit cost. ${ }^{108}$ BIA is limited by its sensitivity to fluid changes, the position in which participants stand and equations for $\% \mathrm{BF}$ and BFM may not be generalisable to all ethnicities. ${ }^{109}$ Further, this study is only conducted at one institution and thus results may not be generalisable to other universities. The single-centre design, however, results in less variability of certain environmental factors including type of cafeteria food, food accessibility and access to physical fitness facilities to be held constant. Finally, the GENEiUS study is not sufficiently powered to find gene-environment interactions which require samples of up to $100000 .{ }^{110}$ Certain subgroup analyses with a low number of individuals, such as Aboriginal Peoples in ethnic-specific analyses, may also be underpowered to find associations. ${ }^{111}$

Overall, the GENEiUS study is the first to investigate the genetic and environmental determinants of weight change in undergraduate students. It will assess a wide range of well-studied and novel potential risk factors. It will contribute to the limited knowledge of weight change throughout undergraduate studies as it will follow participants longitudinally. The prospective nature of this observational study will help to make causal inferences for weight change in undergraduate students. Since the duration of obesity is associated with higher morbidity and mortality, understanding the determinants of weight gain in young adulthood may lead to major improvements in the quality of life and life expectancy, as well as decrease the burden that obesity places on public health infrastructure.

\section{Ethics and dissemination}

Prior to enrolment, a member of the research team will explain the consent form in plain language and each student then will be asked to read and provide written informed consent. The informed consent states the voluntary nature of participation and that 
withdrawal from the study may be done at any time. All methods and procedures for this study (\#0524) were reviewed and approved by the Hamilton Integrated Research Ethics Board. Outcomes for this research will be published in peer-reviewed journals and presented at scholarly regional, national and international meetings. Meetings will occur with university leaders like the associate dean of education, administration and student groups at McMaster University to facilitate knowledge translation to the local stakeholders and student population.

Acknowledgements We would like to extend our thanks to Tooba Fatima, Huphy Ghayur, Danielle Henry, ShawnKhan, Hsin-Yen Liu, Sumiya Lodhi, Zechen Ma, Sarib Malik, Sebat Mohammed, Austin Solak, Emily Tam, Vivian Tam and Christy Yu for their contribution. DM holds a Canada Research Chair in Genetics of Obesity. REM is supported by the Canadian Institutes of Health Research Canada Graduate Scholarship.

Contributors All authors designed the study protocol; REM and DM wrote the manuscript; CL, AA, AFI, FTY, AJM, HR, JM and MP critically reviewed the manuscript for important intellectual content; DM had primary responsibility for final content. All authors read and approved the final manuscript.

Competing interests None declared.

Provenance and peer review Not commissioned; externally peer reviewed.

Open Access This is an Open Access article distributed in accordance with the Creative Commons Attribution Non Commercial (CC BY-NC 4.0) license, which permits others to distribute, remix, adapt, build upon this work non-commercially, and license their derivative works on different terms, provided the original work is properly cited and the use is non-commercial. See: http://creativecommons.org/ licenses/by-nc/4.0/

(C) Article author(s) (or their employer(s) unless otherwise stated in the text of the article) 2017. All rights reserved. No commercial use is permitted unless otherwise expressly granted.

\section{REFERENCES}

1. Finucane MM, Stevens GA, Cowan MJ, et al. National, regional, and global trends in body-mass index since 1980: systematic analysis of health examination surveys and epidemiological studies with 960 country-years and 9.1 million participants. Lancet 2011;377:557-67.

2. Twells LK, Gregory DM, Reddigan J, et al. Current and predicted prevalence of obesity in Canada: a trend analysis. CMAJ Open 2014;2:E18-E26.

3. Guh DP, Zhang W, Bansback N, et al. The incidence of comorbidities related to obesity and overweight: a systematic review and meta-analysis. BMC Public Health 2009;9:88.

4. Luppino FS, de Wit LM, Bouvy PF, et al. Overweight, obesity, and depression: a systematic review and meta-analysis of longitudinal studies. Arch Gen Psychiatry 2010;67:220-9.

5. Janssen I. The public health burden of obesity in Canada. Can J Diabetes 2013;37:90-6.

6. Guo SS, Chumlea WC. Tracking of body mass index in children in relation to overweight in adulthood. Am J Clin Nutr 1999;70:145s-8.

7. Canada S. Body mass index, overweight or obese, self-reported, adult, by age group and sex (Number of persons). Cansim 2016. Catalogue \#82-221-X.

8. Mokdad AH, Serdula MK, Dietz WH, et al. The spread of the obesity epidemic in the United States, 1991-1998. JAMA 1999;282:1519-22.

9. Baum CL. The effects of college on weight: examining the 'Freshman 15' myth and other effects of college over the life cycle. Demography 2017;54:26.

10. McLaren L. Socioeconomic status and obesity. Epidemiol Rev 2007;29:29-48.

11. Crombie AP, llich JZ, Dutton GR, et al. The freshman weight gain phenomenon revisited. Nutr Rev 2009;67:83-94.

12. Vella-Zarb RA, Elgar FJ. The 'freshman 5 ': a meta-analysis of weight gain in the freshman year of college. J Am Coll Health 2009;58:161-6.
13. Vadeboncoeur C, Townsend N, Foster C. A meta-analysis of weight gain in first year university students: is freshman 15 a myth? BMC Obes 2015;2:22.

14. Girz L, Polivy J, Provencher V, et al. The four undergraduate years. Changes in weight, eating attitudes, and depression. Appetite 2013;69:145-50.

15. Hovell MF, Mewborn CR, Randle Y, et al. Risk of excess weight gain in university women: a three-year community controlled analysis. Addict Behav 1985;10:15-28.

16. Gropper SS, Simmons KP, Connell LJ, et al. Changes in body weight, composition, and shape: a 4-year study of college students. Appl Physiol Nutr Metab 2012;37:1118-23.

17. Pope L, Hansen D, Harvey J. Examining the weight Trajectory of college students. J Nutr Educ Behav 2017;49:137-41.

18. Silventoinen $\mathrm{K}$, Rokholm B, Kaprio J, et al. The genetic and environmental influences on childhood obesity: a systematic review of twin and adoption studies. Int J Obes 2010;34:29-40.

19. Kanter R, Caballero B. Global gender disparities in obesity: a review. Adv Nutr 2012;3:491-8.

20. Levitsky DA, Garay J, Nausbaum M, et al. Monitoring weight daily blocks the freshman weight gain: a model for combating the epidemic of obesity. Int J Obes 2006;30:1003-10.

21. Lowe MR, Annunziato RA, Markowitz JT, et al. Multiple types of dieting prospectively predict weight gain during the freshman year of college. Appetite 2006;47:83-90.

22. Pliner P, Saunders T. Vulnerability to freshman weight gain as a function of dietary restraint and residence. Physiol Behav 2008;93:76-82.

23. Lacaille LJ, Dauner KN, Krambeer RJ, et al. Psychosocial and environmental determinants of eating behaviors, physical activity, and weight change among college students: a qualitative analysis. $J$ Am Coll Health 2011;59:531-8.

24. Greaney ML, Less FD, White AA, et al. College students' barriers and enablers for healthful weight management: a qualitative study. $J$ Nutr Edu c Behav 2009;41:281-6.

25. Wansink B, Cao Y, Saini P, et al. College cafeteria snack food purchases become less healthy with each passing week of the semester. Public Health Nutr 2013;16:1291-5.

26. Kasparek DG, Corwin SJ, Valois RF, et al. Selected health behaviors that influence college freshman weight change. J Am Coll Health 2008;56:437-44.

27. Pullman AW, Masters RC, Zalot LC, et al. Effect of the transition from high school to university on anthropometric and lifestyle variables in males. Appl Physiol Nutr Metab 2009;34:162-71.

28. Serlachius A, Hamer M, Wardle J. Stress and weight change in university students in the United Kingdom. Physiol Behav 2007:92:548-53.

29. Deforche B, Van Dyck D, Deliens T, et al. Changes in weight physical activity, sedentary behaviour and dietary intake during the transition to higher education: a prospective study. Int J Behav Nutr Phys Act 2015;12:16.

30. Teixeira PJ, Going SB, Houtkooper LB, et al. Exercise motivation, eating, and body image variables as predictors of weight control. Med Sci Sports Exerc 2006;38:179-88.

31. Taheri S, Lin L, Austin D, et al. Short sleep duration is associated with reduced leptin, elevated ghrelin, and increased body mass index. PLoS Med 2004;1:e62.

32. Boyce JA, Kuijer RG. Perceived stress and freshman weight change: the moderating role of baseline body mass index. Physiol Behav 2015;139:491-6.

33. Cheney AM, Booth BM, Davis TD, et al. The role of borderline personality disorder and depression in the relationship between sexual assault and body mass index among women veterans. Violence Vict 2014;29:742-56.

34. Felitti VJ. Long-term medical consequences of incest, rape, and molestation. South Med J 1991;84:328-31.

35. Fedina L, Holmes JL, Backes BL. Campus sexual assault. Trauma Violence Abuse; 15:152483801663112.

36. Amlung M, Petker T, Jackson J, et al. Steep discounting of delayed monetary and food rewards in obesity: a meta-analysis. Psychol Med 2016;46:2423-34.

37. Murphy CM, Stojek MK, MacKillop J. Interrelationships among impulsive personality traits, food addiction, and Body Mass Index. Appetite 2014;73:45-50.

38. Villarejo C, Fernández-Aranda F, Jiménez-Murcia S, et al. Lifetime obesity in patients with eating disorders: increasing prevalence, clinical and personality correlates. Eur Eat Disord Rev 2012;20:250-4. 
39. Hoek HW. Incidence, prevalence and mortality of anorexia nervosa and other eating disorders. Curr Opin Psychiatry 2006;19:389-94.

40. Lipson SK, Sonneville KR. Eating disorder symptoms among undergraduate and graduate students at 12 U.S. colleges and universities. Eat Behav 2017;24:81-8.

41. Gariepy G, Nitka D, Schmitz N. The association between obesity and anxiety disorders in the population: a systematic review and meta-analysis. Int J Obes 2010;34:407-19.

42. Association ACH. American college health association-National College Health Assessment II: Ontario Canada reference group executive summary spring. Hanover, MD: American College Health Association, 2016.

43. Odlaug BL, Lust K, Wimmelmann CL, et al. Prevalence and correlates of being overweight or obese in college. Psychiatry Res 2015;227:58-64.

44. van den Berg PA, Mond J, Eisenberg M, et al. The link between body dissatisfaction and self-esteem in adolescents: similarities across gender, age, weight status, race/ethnicity, and socioeconomic status. J Adolesc Health 2010;47:290-6.

45. Gillen MM, Lefkowitz ES. Gender and racial/ethnic differences in body image development among college students. Body Image 2012;9:126-30.

46. CNy H, Jackson LA, Sullivan LA. The 'FRESHMAN 15' facts and fantasies about weight gain in college women. Psychol Women $Q$ 1993;17:119-26.

47. Canada S Religion (108). Immigrant Status and Period of Immigration (11), Age Groups (10) and Sex (3) for the Population in Private Households of Canada, Provinces, Territories, Census Metropolitan Areas and Census Agglomerations, 2011 National Household Survey. National Household Survey 2011. Catalogue no. 99-010-X2011032.

48. Rew L, Wong YJ. A systematic review of associations among religiosity/spirituality and adolescent health attitudes and behaviors. J Adolesc Health 2006;38:433-42.

49. Banerjee AT, Boyle MH, Anand SS, et al. The relationship between religious service attendance and coronary heart disease and related risk factors in Saskatchewan, Canada. J Relig Health 2014;53:141-56.

50. Lycett D. The association of religious affiliation and Body Mass Index (BMI): an analysis from the health survey for England. J Relig Health 2015;54:2249-67.

51. Feinstein M, Liu K, Ning $\mathrm{H}$, et al. Incident obesity and cardiovascular risk factors between young adulthood and middle age by religious involvement: the Coronary Artery Risk Development in Young Adults (CARDIA) study. Prev Med 2012;54:117-21.

52. Goel MS, McCarthy EP, Phillips RS, et al. Obesity among US immigrant subgroups by duration of residence. JAMA 2004;292:2860-7.

53. Locke AE, Kahali B, Berndt SI, et al Genetic studies of body mass index yield new insights for obesity biology. Nature 2015;518:197-206.

54. Shungin D, Winkler TW, Croteau-Chonka DC, et al. New genetic loci link adipose and insulin biology to body fat distribution. Nature 2015;518:187-96.

55. Lu Y, Day FR, Gustafsson S, et al. New loci for body fat percentage reveal link between adiposity and cardiometabolic disease risk. Nat Commun 2016;7:10495.

56. Meisel SF, Beeken RJ, van Jaarsveld $\mathrm{CH}$, et al. The association of FTO SNP rs9939609 with weight gain at University. Obes Facts 2015;8:243-51.

57. Dale M. Trends in the Age Composition of College and University Students and Graduates. Education Matters: Insights on Education, Learning and Training in Canada (81-004-X) Vol 7, Statistics Canada; 2010. http://www.statcan.gc.ca/pub/81-004-x/2010005/ article/11386-eng.htm (accessed 23 Aug 2017).

58. Organization WH. Waist circumference and waist-hip ratio: report of a WHO expert Consultation. Geneva: World Health Organization, 2008.

59. Thompson FE, Byers T. Dietary assessment resource manual. J Nutr 1994;124:2245s-317.

60. Subar AF, Kirkpatrick SI, Mittl B, et al. The Automated SelfAdministered 24-hour dietary recall (ASA24): a resource for researchers, clinicians, and educators from the National cancer institute. J Acad Nutr Diet 2012;112:1134-7.

61. Babor TF, Higgins-Biddle JC, Saunders JB, et al. AUDIT: the alcohol use disorders identification test: guidelines for use in primary health care. Geneva: World Health Organization, 2001.

62. Bull FC, Maslin TS, Armstrong T. Global physical activity questionnaire (GPAQ): nine country reliability and validity study. $J$ Phys Act Health 2009;6:790-804.
63. Chu $\mathrm{AH}, \mathrm{Ng} \mathrm{SH}$, Koh D, et al. Reliability and validity of the self- and interviewer-administered versions of the Global Physical Activity Questionnaire (GPAQ). PLoS One 2015;10:e0136944.

64. Organization WH. Global physical activity questionnaire (GPAQ) analysis guide. Geneva: World Health Organization, 2012.

65. Markland D, Tobin V. A modification to the behavioural regulation in exercise questionnaire to Include an assessment of amotivation. $J$ Sport Exerc Psychol 2004;26:191-6.

66. Lund HG, Reider BD, Whiting AB, et al. Sleep patterns and predictors of disturbed sleep in a large population of college students. J Adolesc Health 2010;46:124-32.

67. Buysse DJ, Reynolds CF, Monk TH, et al. The Pittsburgh sleep quality index: a new instrument for psychiatric practice and research. Psychiatry Res 1989;28:193-213.

68. Stalder T, Steudte-Schmiedgen S, Alexander N, et al. Stress-related and basic determinants of hair cortisol in humans: A meta-analysis. Psychoneuroendocrinology 2017;77:261-74.

69. Wennig R. Potential problems with the interpretation of hair analysis results. Forensic Sci Int 2000;107:5-12.

70. Russell E, Koren G, Rieder M, et al. Hair cortisol as a biological marker of chronic stress: current status, future directions and unanswered questions. Psychoneuroendocrinology 2012;37:589-601.

71. Kooiman CG, Ouwehand AW, ter Kuile MM. The Sexual and Physical Abuse Questionnaire (SPAQ). A screening instrument for adults to assess past and current experiences of abuse. Child Abuse Negl 2002;26:939-53.

72. Cyders MA, Littlefield AK, Coffey S, et al. Examination of a short English version of the UPPS-P Impulsive Behavior Scale. Addict Behav 2014;39:1372-6.

73. Whiteside SP, Lynam DR. The Five Factor Model and impulsivity: using a structural model of personality to understand impulsivity. Pers Individ Dif 2001;30:669-89.

74. Koffarnus MN, Bickel WK. A 5-trial adjusting delay discounting task: accurate discount rates in less than one minute. Exp Clin Psychopharmacol 2014;22:222-8.

75. Stice E, Telch CF, Rizvi SL. Development and validation of the eating disorder diagnostic scale: a brief self-report measure of anorexia, bulimia, and binge-eating disorder. Psychol Assess 2000;12:123-31.

76. Pook M, Tuschen-Caffier B, Brähler E. Evaluation and comparison of different versions of the Body Shape Questionnaire. Psychiatry Res 2008;158:67-73.

77. Cooper PJ, Taylor MJ, Cooper Z, et al. The development and validation of the body shape questionnaire. Int $J$ Eat Disord 1987;6:485-94.

78. Rosenberg M. Society and the adolescent self-image. NJ Princeton: Princeton university press, 1965

79. Radloff LS. The CES-D Scale. Appl Psychol Meas 1977;1:385-401.

80. Shean G, Baldwin G. Sensitivity and specificity of depression questionnaires in a college-age sample. J Genet Psychol 2008;169:281-92.

81. Donker T, van Straten A, Marks I, et al. Quick and easy self-rating of generalized anxiety disorder: validity of the Dutch web-based GAD7, GAD-2 and GAD-SI. Psychiatry Res 2011;188:58-64.

82. Koenig H, Parkerson GR, Meador KG. Religion index for psychiatric research. Am J Psychiatry 1997;154:885-6.

83. Koenig HG, Büssing A. The Duke University Religion Index (DUREL): a five-item measure for use in epidemological studies. Religions 2010;1:78-85.

84. Speliotes EK, Willer CJ, Berndt SI, et al. Association analyses of 249,796 individuals reveal 18 new loci associated with body mass index. Nat Genet 2010;42:937-48.

85. Lubitz SA, Brody JA, Bihlmeyer NA, et al. Whole exome sequencing in atrial fibrillation. PLoS Genet 2016;12:e1006284.

86. Dudbridge F, Gusnanto A. Estimation of significance thresholds for genomewide association scans. Genet Epidemiol 2008;32:227-34

87. Manolio TA, Collins FS, Cox NJ, et al. Finding the missing heritability of complex diseases. Nature 2009;461:747-53.

88. Li A, Meyre D. Challenges in reproducibility of genetic association studies: lessons learned from the obesity field. Int $J$ Obes 2013;37:559-67.

89. Yang J, Lee SH, Goddard ME, et al. GCTA: a tool for genome-wide complex trait analysis. Am J Hum Genet 2011;88:76-82.

90. Purcell S, Neale B, Todd-Brown K, et al. PLINK: a tool set for wholegenome association and population-based linkage analyses. Am J Hum Genet 2007;81:559-75.

91. Howie B, Fuchsberger C, Stephens M, et al. Fast and accurate genotype imputation in genome-wide association studies through pre-phasing. Nat Genet 2012;44:955-9. 
92. Howie BN, Donnelly P, Marchini J. A flexible and accurate genotype imputation method for the next generation of genome-wide association studies. PLoS Genet 2009;5:e1000529.

93. Mishra A, Macgregor S. VEGAS2: software for more flexible genebased testing. Twin Res Hum Genet 2015;18:86-91.

94. Li MX, Gui HS, Kwan JS, et al. A comprehensive framework for prioritizing variants in exome sequencing studies of Mendelian diseases. Nucleic Acids Res 2012;40:e53.

95. Price AL, Patterson NJ, Plenge RM, et al. Principal components analysis corrects for stratification in genome-wide association studies. Nat Genet 2006;38:904-9.

96. Dudbridge F. Power and predictive accuracy of polygenic risk scores. PLoS Genet 2013;9:e1003348.

97. Wei Z, Wang K, Qu HQ, et al. From disease association to risk assessment: an optimistic view from genome-wide association studies on type 1 diabetes. PLoS Genet 2009;5:e1000678.

98. Alyass A, Almgren P, Akerlund M, et al. Modelling of OGTT curve identifies $1 \mathrm{~h}$ plasma glucose level as a strong predictor of incident type 2 diabetes: results from two prospective cohorts. Diabetologia 2015;58:87-97.

99. Anand SS, Meyre D, Pare G, et al. Genetic information and the prediction of incident type 2 diabetes in a high-risk multiethnic population: the EpiDREAM genetic study. Diabetes Care 2013;36:2836-42.

100. Barrio I, Arostegui I, Rodríguez-Álvarez M-X, et al. A new approach to categorising continuous variables in prediction models: Proposa and validation. Stat Methods Med Res 2015;52:.

101. Austin PC, Steyerberg EW. Events per variable (EPV) and the relative performance of different strategies for estimating the outof-sample validity of logistic regression models. Stat Methods Med Res 2017;26:796-808.
102. McAllister EJ, Dhurandhar NV, Keith SW, et al. Ten putative contributors to the obesity epidemic. Crit Rev Food Sci Nutr 2009;49:868-913.

103. Rothman KJ. BMI-related errors in the measurement of obesity. Int J Obes 2008;32:S56-9.

104. Dietz WH. Health consequences of obesity in youth: childhood predictors of adult disease. Pediatrics 1998;101:518.

105. Wang YC, McPherson K, Marsh T, et al. Health and economic burden of the projected obesity trends in the USA and the UK. Lancet 2011;378:815-25.

106. Dhuper S, Buddhe S, Patel S. Managing cardiovascular risk in overweight children and adolescents. Paediatr Drugs 2013;15:181-90.

107. Cleland CL, Hunter RF, Kee F, et al. Validity of the global physical activity questionnaire (GPAQ) in assessing levels and change in moderate-vigorous physical activity and sedentary behaviour. BMC Public Health 2014;14:1255.

108. Sun G, French CR, Martin GR, et al. Comparison of multifrequency bioelectrical impedance analysis with dual-energy X-ray absorptiometry for assessment of percentage body fat in a large, healthy population. Am J Clin Nutr 2005;81:74-8.

109. Dehghan $M$, Merchant AT. Is bioelectrical impedance accurate for use in large epidemiological studies? Nutr J 2008;7:26.

110. Reddon H, Guéant JL, Meyre D. The importance of geneenvironment interactions in human obesity. Clin Sci 2016;130:1571-97.

111. Reddon $\mathrm{H}$, Gerstein HC, Engert JC, et al. Physical activity and genetic predisposition to obesity in a multiethnic longitudinal study. Sci Rep 2016;6:18672. 